\title{
Resistencia al cambio en las organizaciones desde la perspectiva del estructuralismo construccionista
}

\author{
Borgucci, Emmanuel*
}

\section{Resumen}

En este artículo se intenta demostrar que en los procesos organizacionales sus actores compiten y establecen relaciones de fuerza con otros individuos con la finalidad de promover unas reglas de juego y un discurso que les aseguren el monopolio del capital organizacional. En tal sentido, se realizó un trabajo de observación participante y de entrevistas en profundidad a un grupo de empleados pertenecientes a una oficina bancaria ubicada en la ciudad de Maracaibo, Estado Zulia. Se concluye que la competencia por el capital organizacional determinó indicios de pérdida inicial del control por parte de la institución bancaria. Por otra parte, al percatarse la empresa de que sus prácticas habituales y discurso eran desafiados, procedió a una reimplantación de los códigos de pertenencia a ese campus por medio de la sustitución del equipo de funcionarios y una reestructuración de carácter operativo y tecnológico sin que eso implicara despido de personal.

Palabras clave: Resistencia al cambio, procesos organizacionales, habitus, campus, discurso gerencial.

\section{Resistance to Change in Organizations from the Perspective of Constructionist Structuralism}

\begin{abstract}
This article intends to demonstrate that in organizational processes, actors compete and establish power relations with other individuals to promote some rules of the game and a discourse that assures them a monopoly of organizational capital. In this light, a study was carried out using participant observation and in-depth interviews with a group of employees belonging to a bank office located in the city of Maracaibo, State of Zulia. Conclusions were that competition for organizational capital determined indexes of initial loss of control by the banking institution. On the other hand, when the

Recibido: 05-03-07. Aceptado: 12-05-08

* Profesor de la Universidad del Zulia, Instituto de Investigaciones Económicas.

E-mail: eborgucci@yahoo.com
\end{abstract}


company realized that its habitual practices and discourse were being challenged, they proceeded to re-implant the codes of belonging to this campus by substituting the team of functionaries and a restructuring of an operational and technological character without implying personnel firing.

Key words: Resistance to change, organizational processes, habitus, campus, managerial discourse.

\section{Introducción}

En la literatura gerencial es muy frecuente encontrar discursos que reclaman, como expresa Robbins: "Hacer que las cosas sean diferentes" (1987:465). El cambio es visualizado como una necesidad para supervivencia de la empresa, la mayor obtención de ganancias, el logro de una mayor participación en el mercado o hacer frente a la volatilidad en el mundo de los negocios. Desde la perspectiva biológica de Hofer y Schendel (1985), a la larga solo sobreviven aquellas organizaciones que sirven a las necesidades de la sociedad efectiva y eficientemente y eso es posible con el "cambio estratégico". Por otra parte, desde el punto de vista económicofinanciero las organizaciones deben, según la teoría de la demanda de activos (Mishkin, 1995), cambiar permanentemente para maximizar la P/E ratio, el margen de utilidad neta y contar con un flujo de caja estable y adecuado. Desde el punto de vista del marketing estratégico y operativo (Barquero et al., 2003; Kotler, 1996; Vassos, 1996; Hill y Jones, 1996; Lambin, 1995), los cambios provienen de la observación permanente de las necesidades de los consumidores en términos de producto, servicios y postventa; además, del ajuste permanente en los análisis de atractivo de mercado y ciclo de vida de los productos. Por último, Lashinsky (2006), expresó citando a Shona Brown, que el camino hacia el éxito organizacional en una era de situaciones ambiguas que se presentan en rápidas etapas requiere evitar crear muchas y grandes estructuras organizacionales (la trampa burocrática que inmoviliza la organización), pero tampoco agregar unas demasiadas pequeñas; es decir, el cambio organizacional consiste en la creación de un ritmo interno de crecimiento sin caer en la trampa del crecimiento. Lo anterior conduce al concepto de creatividad organizacional (Wood- man, Sawyer y Griffin, 1993) que consiste en la creación de productos, servicios, ideas, procedimientos o procesos por individuos trabajando en conjunto en un contexto social complejo.

Lo antes expuesto indica que durante la existencia de las organizaciones, cuando interactúan con su entorno de negocios, son constantemente desafiadas a crear estrategias y prácticas que les permitan sobrevivir y eventualmente liderar el sector económico al que están adscritos. En otras palabras, las organizaciones se encuentran sometidas a una serie de fuerzas de carácter individual, organizacional y societal que promueven prácticas organizacionales tendientes a la búsqueda de cambios en los niveles directivo, operativo y funcional. Sin embargo, existen fuerzas de carácter individual, organizacional y societal que promueven la estabilidad organizacional (Leana y Barry, 2000) y eventualmente la resistencia al cambio. 
Resistencia al cambio en las organizaciones desde la perspectiva del estructuralismo... Borgucci, Emmanuel

En este ensayo, discutiremos cómo la resistencia al cambio se presenta en una organización, concretamente en una agencia bancaria, ubicada en la ciudad de Maracaibo. La resistencia al cambio se enfocará desde la perspectiva de habitus-campus de Bourdieu (2001) y tomando en consideración que en las organizaciones se desarrollan una serie de prácticas que pueden ser entendidas como discursos (Townley, 1993) que intentan reducir la indeterminación dentro en las interacciones en el nivel funcional de la organización.

\section{Condiciones en que se favorece el cambio o la estabilidad organizacional y la resistencia al cambio}

Leana y Barry (2000) identificaron al menos cinco fuerzas que motorizan el cambio en las organizaciones y cinco que motorizan la estabilidad. Las primeras cinco fuerzas se refieren a la no-volatilidad, la contención de costos (gastos), la impaciencia de los mercados, nuevas formas de control organizacional y la búsqueda de ventajas competitivas.

Burns and Stalker (1961) llamaron la atención acerca de la necesidad de establecer estructuras orgánicas en las organizaciones ante contextos volátiles. En aquel momento, la volatilidad estaba asociada a la existencia de mercados estables de productos tradicionales; mientras que hoy en día la volatilidad está asociada además con procesos comerciales, financieros, tecnológicos y culturales. En esta línea de análisis, la estabilidad laboral, de políticas de mercadeo o de producción impide la adaptación de la organización ante un entorno cambiante. Por otra parte, en las economías desarrolladas como aquellas afecta- das por procesos inflacionarios, los propietarios de empresas son cada vez más impacientes acerca del retorno de sus inversiones. La impaciencia de los propietarios se manifiesta en diferentes formas: incluyendo más presión para obtener resultados a los administradores, cambios de administradores, énfasis en un manejo más agresivo de los fondos de tesorería o confundir activos que deben ser gerenciados con costos que deben ser reducidos (Pfeffer, 1998). Lo anterior ha llevado a que se establezcan sistemas de trabajo que garanticen más poder y control sin necesidad de centralizar la organización (Sennett, 1998). Por último, todo lo anterior sugiere que las empresas deben definir, ponderar y hacer efectivas ventajas competitivas.

Las organizaciones promueven la estabilidad principalmente en cómo el trabajo está configurado y cómo es llevado a cabo (Leana y Barry, 2000: 754). En primer lugar, se encuentra la tendencia a hacer las cosas hoy, siguiendo los buenos resultados del pasado. Las organizaciones recurren a los éxitos del pasado no porque sea más fácil sino debido a que no se piensa de manera diferente (Buró, 1992). Además, como lo expresa Pfeffer (1981), las estructuras de poder existentes tienden a autoperpetuarse en términos de relaciones y prácticas. En segundo lugar, se encuentran los efectos de los costos de transacción (Williamson, 1975). Si el sector económico es poco volátil, una solución eficiente consistirá en invertir en el mejoramiento profesional del personal que, a su vez, garantizará ventajas competitivas sostenibles para la empresa. En tercer lugar, la estabilidad organizacional se logra por efectos del 
capital social organizacional (Leana y Van Buren, 1999); es decir, el conjunto de recursos con que cuenta la organización como reflejo de las relaciones sociales de sus miembros y que facilita una acción colectiva exitosa. En consecuencia, como expresaron Leana y Rousseau (2000: 755-756): "La estabilidad y el cambio son ambos necesarios para que la organización funcione efectivamente".

Sin embargo, existe la resistencia al cambio. Robbins y Judge (2006) hablan de dos razones por la que se presenta la resistencia al cambio: la pérdida de lo conocido y la pérdida personal. La primera es la aversión por la aplicación de nuevas reglas de trabajo; la segunda está referida al status quo conquistado. Stoner y Wankel (1989) agregan a los dos anteriores: la incertidumbre de las causas y efectos del cambio y la conciencia de las debilidades en los cambio propuestos. Desde una perspectiva más filosófica, Brown (1992), citando a Kenenth Burke, sugiere que la resistencia al cambio puede provenir de cómo las personas han definido los propósitos que justifican sus actos. En tal sentido Brown (1992) habla de una ética del propósito (meta generada por el agente), una ética del principio (evaluación de los actos en función de reglas) y una ética de la consecuencia (evaluación de los actos en función de resultados probables). Desde el punto de vista del psicoanálisis, los mecanismos de resistencia al cambio cul- tural en una empresa son según García y Dolan (1997): represión (lagunas en la memoria y olvidos), regresión (recurrir a conductas que proporcionaron seguridad en el pasado), proyección (atribución a otra persona de una cualidad que una persona tiene), identificación (con el agente de cambio, no como aceptación del cambio sino como mecanismo de defensa), formación reactiva o mecanismo contrafóbico, la negación o ignorar parte de la realidad no ajustada a los deseos de una persona, la resistencia de beneficio secundario y las del superyó.

No obstante, la resistencia al cambio puede proceder por razones no personales o grupales. Más aún la resistencia al cambio puede provenir de conflictos entre o dentro de diversos grupos del nivel directivo, operativo o funcional. En consecuencia, las organizaciones son la confluencia de estructuras mentales y estructuras objetivas que orientan prácticas sociales gerenciales y laborales.

\section{La noción de habitus y campus desde el estructuralismo constructuvista ${ }^{1}$}

De lo anteriormente expuesto, las prácticas sociales en general y en nuestro caso, las prácticas sociales en un determinado departamento (agencia) de la organización, proviene de la confluencia de estructuras sociales y estructuras

1 Es un intento de Pierre Bourdieu de superar la antinomia entre subjetivismo y objetivismo. Se opone a una concepción totalizante que explique la sociedad como determinante de las acciones de los individuos y se opone además a una concepción en donde la sociedad es producto de la experiencia individual al margen del contexto social. 
Resistencia al cambio en las organizaciones desde la perspectiva del estructuralismo... Borgucci, Emmanuel

mentales. Ambas orientan y condicionan históricamente las prácticas sociales. Para Bourdieu, las estructuras sociales las encuadró dentro del concepto de campus y las segundas dentro de lo que él denominó como habitus ${ }^{2}$. Para Bourdieu (1996) el habitus es:

"... a la vez un sistema de esquemas de producción de prácticas y un sistema de esquemas de percepción y de apreciación de las prácticas. Y, en los dos casos, sus operaciones expresan la posición social en la cual se ha construido. En consecuencia, el habitus produce prácticas y representaciones que están disponibles para la clasificación, que están objetivamente diferenciadas; pero no son inmediatamente percibidas como tales más que por los agentes que poseen el código, los esquemas clasificatorios necesarios para comprender su sentido social" (1996: 134).

En otras palabras, el habitus desde el punto de vista del sujeto, es un conjunto de esquemas clasificatorios que son socialmente impuestos y que dependen de sus condiciones de existencia. Esas condiciones de existencia están constituidas por prácticas que, a su vez, están organizadas por configuraciones sistemáticas de propiedades que expresan sistemas de valo- res diferenciales. Además, según Bourdieu los actores sociales: “. . . son capaces de ver la relación entre las prácticas o las representaciones y las posiciones en el espacio social. Así, a través del habitus, tenemos un mundo del sentido común. .." (1996: 135). Además el espacio social, gracias a la presencia de actores sociales con propiedades diferenciales (con poder de diferenciación) y ligados sistemáticamente, da lugar a grupos de interacciones de diferente categoría y que responden a diferentes principios de visión y división. De esta manera las prácticas sociales no se presentan como totalmente estructuradas y, por lo tanto, capaces de imponer a todos los actores sociales los principios de su construcción.

En resumen, el concepto de habitus en Pierre Bourdieu implica:

1. Que las percepciones y representaciones no son libremente motivadas. El autor además agrega, que el habitus se adquiere inconscientemente, pero no es el inconsciente. No es mecánico o espontáneo, debido a que no está estructurado según una lógica de referencia a un fin.

2. Proviene de un proceso de inculcación que transforma lo cultural en natural. En tal sentido, el autor señala en el proceso de inculcación se genera

2 Para Bourdieu (2001), el habitus son estructuras constitutivas de un "entorno", son lo que él denomina como "regularidades asociadas", que constituyen un sistema de disposiciones duraderas de acciones organizadas por actores sociales y son estructuras estructuradoras que funcionan como estructuras estructurantes de prácticas sociales. El concepto de habitus en Bourdieu es similar al de figuración de Norbert Elias (1995). La figuración son formas de pensamiento, actuación e interacción en un momento histórico determinado y que definen lo normal y lo anormal. Es decir, en la estructura social se presentan condiciones de posibilidad y limitaciones a la interdependencia de los individuos. 
un habitus primario proveniente del entorno familiar (disposiciones duraderas y no inmutables que condicionan la adquisición de nuevas disposiciones y la realización de prácticas), un habitus secundario (que se constituye en la escuela) y un habitus de clase (es el habitus individual que refleja el del grupo).

3. La transferibilidad del habitus da origen a un principio generador de prácticas y de enjuiciar las prácticas sociales que permiten actuar al habitus en el tiempo. Por lo tanto, ante situaciones cambiantes en el contexto social, se produce el efecto histéresis. Es decir, aunque desaparezca las condiciones objetivas que permitieron reproducir prácticas socialmente válidas, se produce un desajuste (décalage) entre el principio que permitió enjuiciar las prácticas pretéritas y el contexto presente.

4. Existe un conjunto de disposiciones perdurables que adaptan las percepciones, pensamientos y acciones de los actores sociales a determinadas situaciones.

5. Tales disposiciones afectan y estructuran diferentes campos de actividad. Esto ocurre debido a que las propiedades diferenciales de los actores sociales distribuidos en el espacio social se expresan en signos distintivos y signos de distinción. Por lo tanto el espacio social es de carácter simbólico en donde se pueden presentar consensos o disputas simbólicas.

Con referencia al escenario donde opera el habitus, Bourdieu (1996) lo denomina campus. El campo son los espacios de juego históricamente constituidos con su estructura de actores, relaciones, instituciones específicas y leyes de funcionamiento. Bourdieu (2001) subdivide el espacio social en un conjunto de campos sociales (microcosmos) vinculados por sistemas de relaciones y sistema de posiciones. En forma resumida, el campo social se estructura y funciona de la siguiente manera:

1. Son espacios estructurados de posiciones de un conjunto de actores sociales (agentes) con una situación actual y potencial. En la sociedad existirán tantos campus como áreas de la cultura humana (se habla de un campus científico, literario, económico o político entre otros);

2. Por otra parte, dentro de cada campo existen grupos que: “... tienen sus dominantes, y sus dominados, sus conservadores y su vanguardia, sus luchas subversivas y sus mecanismos de reproducción" (Bourdieu, 1996: 143). Es decir, los actores sociales compiten con su capital (económico, político, social, cultural o simbólico) para establecer las reglas y códigos que aseguran la posesión y acceso al capital o poder que regulan al campo. Además, el autor utiliza la metáfora del mercado para detallar el tipo de actor involucrado. Por una parte se encuentran los productores, los consumidores y los mediadores que representan las instancias de consagración y legitimación específica del campo.

3. El control sobre las formas de capital, reglas de juego y/o códigos permiten establecer las normas de adhesión y poder motivador (investissement) que impulsan la acción. Se trata de una participación de un juego estraté- 
Resistencia al cambio en las organizaciones desde la perspectiva del estructuralismo... Borgucci, Emmanuel

gico. De esta manera, cada campo engendra su interés (illusio) que le es propio y cada interés es la condición de funcionamiento del campo. Es decir, que los actores sociales dentro de un campo determinado están de acuerdo en que lo sucedido dentro del campo tiene sentido, que sus apuestas como jugadores son importantes. Por lo tanto, los actores estarán dispuestos a luchar dentro del campo por lo que en ese campo se lucha y obtener beneficios (que pueden ser de carácter económico o simbólico) de ello.

4. Cada campus se organiza según el capital obtenido por las personas y/o grupos que lo componen en forma coaligada o no en luchas anteriores y que orienta la estrategia de los actores comprometidos. Es decir, dentro de cada campus, los actores sociales cuentan con diferentes formas de capital de tipo económico (bienes y servicios), cultural (conocimiento adquirido por medio del sistema educativo) o simbólico (a través del prestigio y la credibilidad).

5. En cada campus se presentan tensiones de campo y luchas de poder por la distribución de las formas de capital y las prácticas sociales "adecuadas". Tales tensiones, en algunos momentos puede favorecer a alguno de los actores involucrados en un momento determinado y desfavorecerlo en otro momento. Sin embargo la acumulación de capitales simbólicos, políticos - económicos favorecerá a quien haga mejor uso de ellos. Lo anterior es así porque el campo es el escenario de luchas destinadas a conservar o transformar ese campo de fuerzas.
En él, los que han acumulado más capital adoptan estrategias de ortodoxia y los que poseen menos capital tratan de aplicar estrategias de herejía (Gutiérrez, 2002). En otras palabras, en cada campus existen agentes poseedores mayoritarios del capital, existen agentes autorizados a emplear el capital del agente principal y existe un conjunto de agentes mas o menos separados que compiten por la posesión del capital.

6. Según Gutiérrez (2002), para que funcione el campo se requiere gente que esté dispuesta a jugar el juego que es propio del campo, que esté dotada del habitus (conocimiento y reconocimiento de las leyes inmanentes del juego, que crea en el valor de lo que en el campo está en juego). La creencia no es voluntaria, sino de una adhesión inmediata e incondicional.

7. Por lo antes expuesto, el campo es dinámico. En él se producen constantes definiciones y redefiniciones de las relaciones de las fuerzas entre los actores comprometidos en el juego. Es decir, a medida en que los actores sociales modifican parcial o sustancialmente sus representaciones sociales, también cambiaran el sentido en que interactúan las diversas fuerzas comprometidas dentro del campo.

8. Los límites entre los diferentes campus sociales son de carácter legal o con barreras de entrada explícitas o tácitas. Por ejemplo, Bourdieu (2001) sugirió la posibilidad de relaciones entre el campus social y el campus del poder, debido a que este último es el lugar en donde las relaciones de fuerzas de diferentes formas de poder luchan por el principio de "domi- 
nación dominante" (Bourdieu, 2001) o de dominación legítima.

Dentro del campus tienen lugar luchas simbólicas por la producción del sentido común, es decir, por alcanzar el monopolio de la nominación legítima de la experiencia. Esa lucha simbólica tiene dos aspectos: de carácter objetivo y de carácter subjetivo. En el primer caso, consiste en actuar por medio de representaciones para hacer ver y valer que ciertas manifestaciones, situaciones y decisiones sean de carácter asertórico. En el segundo caso, existe una lucha por cambiar las categorías de percepción y apreciación de la experiencia, por medio de la introducción de estructuras cognitivas y evaluativas.

El resultado de la lucha simbólica dentro de cada campus, luego que los actores sociales involucrados en las luchas simbólicas aplican sus formas de capital adquirido, es que algún grupo o coalición de grupos legitimen cierto orden social, es decir, la aplicación de representaciones sociales que promuevan estructuras de percepción y apreciación. Tales representaciones deben propiciar categorias inherentes y juicios asertóricos (incluso apodícticos por medio del efecto teoría, es decir, la capacidad de una representación o discurso de ser útil, adecuado o verificable) con la finalidad de crear en las personas auténticas creencias.

Resta solamente el proceso de conversión de un discurso no oficial en un discurso oficial sobre la base de las nue- vas representaciones sociales y estructuras de percepción y evaluación vigentes. Este proceso, según Bourdieu (1999) se desarrolla por medio de los que él denominó como proceso de "inculcación simbólica" o de "impregnación gota a gota". De esta manera, el discurso oficial adquiere un poder simbólico oficial.

Lo anteriormente señalado, conduce a lo que Bourdieu denominó como campus jurídico, que lo definió como: “...el lugar de una concurrencia por el monopolio del derecho de decir el derecho, esto es, la buena distribución (nomos) o el buen orden en el que se enfrentan agentes investidos de una competencia inseparablemente social y técnica, que consiste esencialmente en la capacidad socialmente reconocida de interpretar (de manera más o menos libre o autorizada) un corpus de textos que consagran la visión legítima, recta, del mundo social" (2001: 169).

Bourdieu (2001) caracterizó a este campus como la de un universo relativamente independiente (y neutral) de prácticas externas y que ejerce una violencia simbólica legítima por medio de la razón jurídica. Es decir, la razón jurídica es la representación recta (droite) de esquemas acordes con las estructuras del mundo que son el producto de consagrar un determinado orden establecido.

Ahora bien como discurso, en el campus jurídico se asegura una unidad de significación por medio de prácticas enunciativas $^{3}$ y el poder de nombramiento que

3 Según Bourdieu (2001), el lenguaje jurídico se produce por la concurrencia de dos efectos: El efecto neutralización y el efecto universalización. El efecto neutralización se obtiene por medio del uso de instrumentos sintácticos tales como la voz pasiva y los giros impersonales. El efecto 
Resistencia al cambio en las organizaciones desde la perspectiva del estructuralismo... Borgucci, Emmanuel

asegura el reconocimiento de la visión dominante como objetiva. Como espacio, es el conjunto de órganos especializados (organizados según una estricta jerarquía) que sirven de soporte al campus que negocia el discurso jurídico y donde se ejerce el poder simbólico por antonomasia.

De esta forma, se establece una relación entre la norma jurídica y las prácticas sociales. La norma opera como un principio de determinación de las prácticas y que traduce en forma simbólica lo que existe en forma de habitus. Es decir, la razón jurídica somete una situación eventualmente poco conveniente para los agentes que detentan en monopolio del poder legítimo a límites codificados positivamente. Así el interés por obedecer (aceptar o adherirse) la norma es mayor al interés en desafiarla.

Además de lo anteriormente expuesto, la eficacia del campus jurídico radica en que combina la lógica política (donde se establece la relación aliados versus rivales con exclusión de un árbitro) y la lógica del campus científico (cuyo principio rector, en este caso, es la lógica del deber-ser positivo), lo que le confiere un poder arbitral avalado por el Estado.

En conclusión, las relaciones de poder tienden a reproducirse en las relaciones de poder simbólico oficial por medio de la adquisición de títulos de propiedad simbólica. Estos títulos no solamente otorgan derechos, ventajas o reconocimientos sino que acrecientan el capital simbólico del grupo y refuerzan la eficacia simbólica del poder simbólico establecido.

Visto así esta formulación de carácter operativo busca destacar:

1. La articulación entre lo individual y lo social, entre las estructuras sociales que condicionan y limitan la acción individual y la persona.

2. No se trata solamente que ciertos conceptos científicos formen parte del conocimiento de sentido común con que las personas representan el medio en el que viven y guían su acción. Es decir, también es posible la construcción y circulación de representaciones sociales en medios en donde se toman decisiones de gran importancia o en donde se requiere un nivel de formación especializada.

3. La lucha por imponer sus discursos como aquellos que son portadores del sentido válido o adecuado. Para esto se precisa desarrollar toda una serie de procesos que implican su circulación y reconocimiento en un contexto específico.

4. En la medida que, dentro de un campus determinado, ciertas representaciones sociales han obtenido el suficiente reconocimiento para estar en condiciones de imponerse por sobre otras representaciones, debido a su capacidad de adecuarse a las necesidades (científicas, políticas o económicas) de los grupos en disputa, se incrementa su eficacia simbólica pro-

universalización se obtiene a través de: el uso de verbos en modo indicativo para enunciar las normas, los verbos conjugados en tercera persona del singular, el uso del pretérito perfecto y el uso de pronombres o adjetivos indefinidos. 
duciéndose lo que Bourdieu (1996) denominó como efecto teoría.

\section{Aspectos de carácter metodológico}

El desarrollo de este estudio se realizó en una oficina bancaria ubicada en la calle 77 en la ciudad de Maracaibo y perteneciente a un banco comercial con sede en la capital de la República concretamente el Banco de Venezuela. Sin embargo, se prefirió no identificar con nombre y apellido los integrantes de la institución por las siguientes razones: 1) En lugar de estudiar personas en particular, se prefirió estudiar procesos, debido a que las relaciones de poder entre los diferentes actores es de carácter relacional; 2) Las prácticas gerenciales y laborales permiten descubrir lo que Foucault (1997) denominó como "el régimen de la verdad" que es propio de ese espacio laboral; 3) Lo importante no es quién ejerce el poder y quienes lo desafían, sino cuáles son las prácticas, técnicas y procedimientos utilizados tanto por quienes ejercen la autoridad como por quienes intentan establecer patrones diferentes de adhesión. Este punto de vista se encuentra en sintonía con el concepto foucaultiano de podersaber (Townley, 1993). Es decir, la gerencia como cualquier forma de ejercer un determinado poder en la sociedad debe ser precedido por alguna forma de racionalidad (la gerencia requiere de un léxico, formas de representar eso que debe ser gerenciado, formas de establecer el orden, mecanismo de supervisión y administración de los empleados y/o grupos de empleados) (Townley,
1993: 520). Pero además de lo antes mencionado, se incluyen mecanismos de adscripción, mecanismos de registro, formas de observar y formas de codificar aquellas conductas individuales o grupales que se quiere mantener dentro de determinados límites de tolerancia organizacional.

Un estudio de estas características sugiere, a nuestro entender, la utilización de métodos cualitativos de investigación. Algunas de las razones que justifican el uso de métodos y técnicas de carácter cualitativo fueron: 1) Las estadísticas sobre improductividad de la agencia solamente hacían referencia a una falta de productividad en el trabajo, pero dejaba por afuera las razones extraoperacionales de la labor en esa agencia desempeñada; y 2) Aunque había indicios de resistencia al cambio, también existían indicios no inmediatamente manifiestos de conductas no vinculadas estrechamente con la resistencia al cambio.

Lo anterior llevó a establecer, en primer lugar, un panorama general del nivel de la organización que se desea observar (identificación de la ubicación organizacional de la oficina, las normas y procedimientos operativos que la rigen así como el conocimiento de las funciones de la nómina) (Cáceres, 1998).

En segundo lugar, realizar una labor de campo, entrando en contacto con las diferentes personas que conformaban el equipo de trabajo de la oficina con la finalidad de construir un cuadro de vida de los personajes en diferentes situaciones de trabajo. Para realizar esta labor, se apeló a la técnica de observación no participante con un instrumento de registro de especímenes (Hurtado, 1998). Se pro- 
Resistencia al cambio en las organizaciones desde la perspectiva del estructuralismo... Borgucci, Emmanuel

cedió al registro de la mayor cantidad de eventos de interacción posibles (reuniones, conversaciones en el cierre al mediodía y/o después de procesar los cheques de la cámara de compensación, en la mañana antes de abrir las operaciones al público, en las reuniones con el gerente, concretamente los días 24 y 31 de diciembre en la mañana, reuniones con el sindicato y el día de las secretarias entre otras situaciones) entre los empleados de un mismo grupo, entre los diferentes grupos y los empleados (individualmente o en grupos) con la plana directiva de la agencia. Debido a lo aleatorio del surgimiento de las situaciones de interacción, se procedió, previa autorización de las personas, al uso de grabadoras y el permitir el registro manual de ciertas situaciones que podrian ser decisivas en la interpretación posterior.

En tercer lugar, se procedió a realizar entrevistas de tipo inestructurada de carácter no dirigido (Hurtado, 1998) para que las personas puedan expresar sus ideas, opiniones y sentimientos respecto a las relaciones con sus compañeros y el grupo directivo de la agencia.

En cuarto lugar, sobre los diversos materiales obtenidos, se trató de establecer las líneas principales del conflicto entre la gerencia antes de su cambio definitivo y los empleados de la agencia con la vieja gerencia y los nuevos gerentes. Lo anterior se realizó mediante la trascripción de las grabaciones, su organización cronológica y su posterior interpretación en función de las intenciones, el significado y la función que determinadas acciones tienen para los personajes y grupos involucrados (Martínez, 1989).

\section{El habitus y campus en una oficina bancaria}

El campo de la organización es un tipo determinado de campo que se encuentra conformado por un conjunto de microcosmos conectados por sistemas de relaciones y sistemas de posiciones. Dentro de cada microcosmos (departamentos funcionales, divisiones, entre otros esquemas organizacionales) entran en juego una serie de actores (potenciales y actuales) con una serie de capitales de carácter económico, social y cultural. Los agentes compiten con su capital y establecen relaciones de fuerzas con los demás agentes. Esas relaciones de fuerzas son la expresión de la intención de los agentes de hacer valer sus reglas y códigos con la finalidad de asegurar su acceso y posesión al capital que es propio de ese campo social. De esta manera, entre el actor poseedor del capital y que establece las reglas y códigos escritos o no de adhesión, existe un conjunto de agentes que compiten por acceder a la posibilidad de generar condiciones de pertenencia al campo y de generar un discurso que justifique un estado de cosas en que cualquier modificación a esas condiciones necesariamente implique procesos de negociación.

En el caso particular de la organización que se estudia, existe una estructura organizacional previamente establecida por manuales de organización y métodos elaborados por la Dirección General del Instituto Bancario. Su elaboración siguió un patrón determinado en la lógica de algunos de los siguientes procesos y procedimientos: 
1. La organización lineal y una serie de funciones, procesos y procedimientos establecidos, que generan una división precisa del trabajo entre jefes y subordinados.

2. Por otra parte, esta estructura esta refrendada por el contrato colectivo entre el Instituto Bancario y su sindicato de trabajadores (Ver Cuadro 1).

3. Normas y procedimientos de auditoria.

4. Normas y procedimientos de seguridad, de los equipos, cajeros peatonales, cajeros automáticos, aperturas en días feriados y fines de semana.

5. Auditoria por operaciones de cargo y abono por medio de las cuentas transitorias, la contabilidad en línea y el denominado granero de la oficina (efectivo en las bóvedas).

6. Normas y procedimientos de auditoria electrónica para tarjetas de crédito y tarjetas de débito.

7. Procedimientos y normas de seguridad industrial al momento de la apertura de la oficina, encendido de los equipos, apertura de las bóvedas, comunicación con la sala de control de operaciones, arqueo de los cajeros, revisión de los cofres de los cajeros, controles de rutina de la labor de los vigilantes, encendido de las cámaras de seguridad. Por otra parte, se encuentran los procedimientos de cierre que incluye: cuadre contable de las operaciones de cargo y abono en todas sus modalidades (hoja de prueba), cuadre del efectivo, cuadre de los cheques de cámara de compensación, comparación entre los saldos de la hoja de prueba, con la hoja de efectivo y los terminales, almacenaje del material controlado (chequeras, libretas de ahorro, tarjetas de débito, estados de cuenta, etc.), apagado de los equipos, cambio de las combinaciones y cierre de las bóvedas, procedimientos de apagado de los equipos de computación, envío de la contabilidad en línea, elaboración de los asientos transitorios, elaboración de las remesas de dinero.

8. Procedimientos de caída de línea.

9. Procedimientos de encendido, apagado y control de los sistemas de comunicaciones (telex), control de las ordenes de pagos telegráficas y del control de ordenes transmitidas y recibidas. Este sistema de comunicaciones se establece debido a que existen oficinas ubicadas en zonas remotas.

10. Normas y procedimientos de posteo de operaciones por los terminales. Eso incluye: los no acepta débito, los bloqueos de cheques de 24 y/o 48 horas, cheques con condiciones especiales, cuentas nóminas, cuentas sobregiradas, pase de la llave de seguridad para operaciones con montos que sobrepasan los límites máximos.

11. Normas y procedimientos de operaciones de crédito. Estas operaciones incluyen: tramitación de solicitudes, solicitud por parte del banco de las garantías mínimas, saldos compensatorios, trámites ante el comité de crédito de la oficina, procedimientos de liquidación, cobro de intereses, cargo en cuentas del cliente. Además se encuentra el manejo de ciertas operaciones como Fideicomiso, Cuantas de Activos Líquidos, entre otras. 
Resistencia al cambio en las organizaciones desde la perspectiva del estructuralismo... Borgucci, Emmanuel

\section{Algunos aspectos de la contratación colectiva entre el banco y sus trabajadores}

\begin{tabular}{|c|c|}
\hline Aspectos del contrato & Condiciones respecto al último contrato \\
\hline Préstamo de vivienda & $\begin{array}{l}\text { 1. Se duplica el monto prestado respecto a lo anterior. } \\
\text { 2. Se reduce de } 6 \text { a } 5 \text { años la antigüedad para solicitar financiamiento. } \\
\text { 3. Tasas de interés inferiores a la tasa pasiva }\end{array}$ \\
\hline $\begin{array}{l}\text { Póliza de hospitalización cirugía } \\
\text { y maternidad }\end{array}$ & 1. La empresa aporta un $20 \%$ adicional. \\
\hline Prima por hijo & 1. Se triplicó. \\
\hline Pensión mínima & 1. De 30 se lleva a 60 días de aguinaldo. \\
\hline Primas de matrimonio & 1. Se eleva un $120 \%$. \\
\hline Bonificación por antigüedad & $\begin{array}{l}\text { Incrementos del } 50 \%, 20 \%, 33,33 \% \text { y del } 100 \% \text { para trabajadores con } 15 \text {, } \\
25,35 \text { años de labor y con } 10,20,30 \text { y } 40 \text { años respectivamente. }\end{array}$ \\
\hline Uniformes & 1. La empresa aporta el $70 \%$ de su valor. \\
\hline Bonificación para cobradores & 1. Se incrementó un $180 \%$. \\
\hline Gastos de transporte & 1. Se incrementó un $400 \%$. \\
\hline Gastos de alimentación & 1. Se incrementó un $330 \%$. \\
\hline Becas & $\begin{array}{l}\text { 1. Los aumentos son de la siguiente forma. } \\
\text { Del } 75 \% \text { para primaria } \\
\text { Del } 50 \% \text { para educación media } \\
67 \% \text { para educación superior }\end{array}$ \\
\hline Útiles escolares & 1. Se incrementó en promedio un $260 \%$. \\
\hline Bonificación para cajeros & 1. Se incrementó un $300 \%$. \\
\hline Bonificación por guardias de cajeros & 1. Incremento del $100 \%$ \\
\hline Horas extras & $\begin{array}{l}\text { 1. } 70 \%, 90 \% \text { y } 110 \% \text { del sueldo diario para horas diurnas, nocturnas } \\
\text { y feriados. }\end{array}$ \\
\hline Bonificación por nacimiento de hijo & 1. Incremento del $100 \%$. \\
\hline Salario mínimo & $\begin{array}{l}\text { 1. } 100 \% \text { de incremento por encima de los que el banco aumenta sobre } \\
\text { la base de los decretado por ley }\end{array}$ \\
\hline Utilidades & 1. 80 días en noviembre, 30 días en diciembre y 15 días en julio \\
\hline Aumentos de sueldo & $\begin{array}{l}\text { 1. Un } 29 \% \text { con la firma del contrato, un } 15 \% \text { el primer año y un } 13 \% \\
\text { en el segundo año. }\end{array}$ \\
\hline Plan de jubilación & $\begin{array}{l}\text { 1. En caso de fallecimiento del jubilado, los herederos recibirán la pensión } \\
\text { hasta por } 2 \text { años. }\end{array}$ \\
\hline Vacaciones & $\begin{array}{l}\text { 1. Entre } 1 \text { a } 10 \text { años, } 20 \text { días y un día adicional por cada año de trabajo } \\
\text { hasta } 10 \text { días. } \\
\text { 2. Entre } 10 \text { y más años, } 20 \text { días hábiles bancarios, } 1 \text { día más por cada año } \\
\text { de servicio. }\end{array}$ \\
\hline Bonificación por tiempo de servicio & $\begin{array}{l}\text { 1. De } 1 \text { a } 3 \text { años: } 25 \% \text {, 4to año: } 35 \% \text {, 5to año: } 45 \%, 6 \text { to año: } 55 \%, 7 m o \\
\text { año: } 65 \% \text {, 8vo año: } 75 \% \text {, 9no año, } 85 \% \text {, } 10 \text { mo año: } 95 \% \text {, y } 11 \text { mo año: } \\
\text { 100\%. }\end{array}$ \\
\hline
\end{tabular}

Fuente: Entrevistas con los empleados y funcionarios de la oficina bancaria. 
Por otra parte, el personal de oficiales, subgerentes y el gerente de la oficina están sometidos por lo dispuesto en la Ley Orgánica del Trabajo cuando se refiere a los denominados empleados de dirección ${ }^{4}$.

En la oficina, existe una serie de cargos ocupados por el personal que van desde la atención al público (Front office) y los empleados que realizan las operaciones internas (Back office) (ver Cuadro 2).

Dentro del conjunto de empleados (32 personas) existían una serie de grupos definidos, los cuales se pueden agrupar de la siguiente forma:

1. El grupo de los pruebistas.

2. El grupo de los terminalistas.

3. El grupo de los cajeros junto al cajero principal y el empleado del aseo.

4. El grupo de atención al público, banca privada y operaciones de crédito.

5. El grupo de subgerentes junto al gerente de la agencia.

El primer grupo era el encargado de verificar que las operaciones de cargo y abono en efectivo por los cajeros cuadren. Lo mismo con el resto de operaciones por cuenta de ahorro, corriente, cheques de gerencia, ingresos y gastos. La lógica de su trabajo radica en el principio de que el balance de comprobación de la oficina debe estar cuadrado todos los días. Por otra parte, los pruebistas deben elaborar lo siguiente: 1) Dos ordenes internas abonando y cargando a efectivo por concepto de pagos realizados y recibidos en el día; 2) Dos avisos de asiento con cargo y abono a la cuenta transitoria operaciones de cuentas corrientes procesadas por terminal (en tales transitorias se registra la sumatoria de las operaciones realizadas cargando y abonando cuentas corrientes y que debe cuadrar con su respectiva cuenta transitoria que recoge en una sola cifra el total de operaciones realizadas en la oficina en ese día); 3) Dos avisos de asiento con cargo y abono a la cuenta transitoria operaciones de cuentas de ahorro procesadas por terminal (en tales transitorias se registra la sumatoria de las operaciones realizadas cargando y abonando cuentas de ahorro y que debe cuadrar con su respectiva cuenta transitoria que recoge en una sola cifra el total de operaciones realizadas en la oficina en ese día).

La verificación de cada transacción se hace magnetizando cada comprobante en una máquina contadora y verificadora de transacciones denominada máquina de prueba. En esa máquina el operador debe postear un código de transacción correspondiente a un cargo y otro código para su correspondiente abono. De

4 Es el empleado que toma decisiones u orientaciones de la empresa, así como el que tiene el carácter de representante del patrono frente a otros trabajadores o terceros y puede sustituirlo, en todo o en parte, en sus funciones. Por otra parte, en ocasiones y conjuntamente, puede ser considerado como trabajador de confianza y de inspección. En el primer caso, su labor implica el conocimiento personal de secretos de funcionamiento de determinados sistemas y procedimientos de seguridad y de carácter comercial de la empresa. en el segundo caso, los oficiales, subgerentes y el gerente de la oficina tienen la potestad dada por la empresa de inspeccionar y vigilar el trabajo de otros trabajadores o el resguardo y seguridad de los bienes de la empresa. 
Resistencia al cambio en las organizaciones desde la perspectiva del estructuralismo... Borgucci, Emmanuel

Cuadro 2

Composición de la nómina de empleados de la oficina bancaria 5 de julio Maracaibo.

\begin{tabular}{|c|c|c|c|c|}
\hline No. & $\begin{array}{l}\text { Apellidos- } \\
\text { Nombres }\end{array}$ & Profesión & Cargo & observaciones \\
\hline 1 & E1 & 3ro de bachillerato & Prueba & 1. Único cargo desde que ingresó. \\
\hline 2 & E2 & 3ro de bachillerato & Secretaria & 1. Secretaria de crédito. \\
\hline 3 & E3 & Bachiller & Oficinista I & $\begin{array}{l}\text { 1. Curso de secretariado y computación } \\
\text { 2. Curso de legislación laboral... }\end{array}$ \\
\hline 4 & E4 & 3ro de bachillerato & Gobierno & 1. Curso de atención al público \\
\hline 5 & E5 & Estudios técnicos & Cajero & $\begin{array}{l}\text { 1. Experiencia en prueba, Ctas. Ctes. } \\
\text { y cambio }\end{array}$ \\
\hline 6 & E6 & Bachiller & $\begin{array}{l}\text { Cajero } \\
\text { principal }\end{array}$ & 1. Único cargo desde que ingreso. \\
\hline 7 & E7 & TSU & $\begin{array}{l}\text { Banca } \\
\text { privada }\end{array}$ & $\begin{array}{l}\text { 1. Verificadora (cursos de grafotécnia) } \\
\text { 2. Experiencia en banca privada } \\
\text { 3. Cursos de atención al público }\end{array}$ \\
\hline 8 & E8 & TSU & Oficinista I & 1. Único cargo desde que ingresó. \\
\hline 9 & E9 & Secretariado & Terminalista & 1. Único cargo desde que ingresó. \\
\hline 10 & E10 & Bachiller & Oficinista & 1. Único cargo desde que ingresó. \\
\hline 11 & E11 & Bachiller & Oficinista & 1. Curso de procesamiento de datos. \\
\hline 12 & E12 & Bachiller & Cajero & 1. Curso de atención al público. \\
\hline 13 & E13 & 3ro de bachillerato & Oficinista & $\begin{array}{l}\text { 1. Curso de instrumentos negociables. } \\
\text { 2. Cursos de cajero y depósito. } \\
\text { 3. Atención al público. } \\
\text { 4. Contabilidad básica. }\end{array}$ \\
\hline 14 & E14 & Bachiller mercantil & Cajero & 1. Único cargo desde que ingresó. \\
\hline 15 & E15 & Secretariado & Oficinista & 1. Único cargo desde que ingresó. \\
\hline 16 & E16 & Secretariado & Oficinista & 1. Experiencia en cuentas de ahorro. \\
\hline 17 & E17 & Bachiller & Terminalista & 1. Único cargo desde que ingresó. \\
\hline 18 & E18 & Bachiller & Oficinista I & $\begin{array}{l}\text { 1. Conformación de cheques. } \\
\text { 2. Curso de terminalista. } \\
\text { 3. Curso de verificación }\end{array}$ \\
\hline 19 & E19 & Bachiller & Prueba & $\begin{array}{l}\text { 1. Experiencia en prueba } \\
\text { 2. Principios básicos de la banca }\end{array}$ \\
\hline 20 & E20 & TSU & Oficinista I & 1. Cursos de computación. \\
\hline 21 & E21 & TSU & Cajero & $\begin{array}{l}\text { 1. Cursos de secretariado y atención } \\
\text { al público. }\end{array}$ \\
\hline 22 & E22 & Bachiller & Oficinista I & 1. Curso de pintura. \\
\hline
\end{tabular}




\section{Cuadro 2. (Continuación)}

\begin{tabular}{|c|c|c|c|c|}
\hline No. & $\begin{array}{l}\text { Apellidos- } \\
\text { Nombres }\end{array}$ & Profesión & Cargo & observaciones \\
\hline 23 & E23 & Bachiller & Cajero & $\begin{array}{l}\text { 1. Cursos de grafotécnia, Fideicomiso } \\
\text { 2. Cursos de prueba, terminalista. } \\
\text { 3. Auditoria computarizada } \\
\text { 4. Contabilidad básica, caja, depósito } \\
\text { y crédito. }\end{array}$ \\
\hline 24 & E24 & Economista & Crédito & $\begin{array}{l}\text { 1. Cursos de mercado de capitales. } \\
\text { 2. Cursos de evaluación de proyectos. } \\
\text { 3. Inglés. }\end{array}$ \\
\hline 25 & E25 & Economista & Sub-gerente & 1. Cursos de computación. \\
\hline 26 & E26 & TSU & Sub-gerente & $\begin{array}{l}\text { 1. Cursos de computación. } \\
\text { 2. Técnicas de supervisión. } \\
\text { 3. Cursos de caja y depósito. }\end{array}$ \\
\hline 27 & E27 & Economista & Gerente & 1. Experiencia como pruebista y cajero. \\
\hline 28 & E28 & Bachiller & Cajero & 1. Presidente del sindicato del banco. \\
\hline 29 & E29 & Bachiller en farmacia & Cajero & 1. Curso de caja y depósito. \\
\hline 30 & E30 & Bachiller & Cajero & $\begin{array}{l}\text { 1. Cursos de contabilidad y atención } \\
\text { al público. }\end{array}$ \\
\hline 31 & E31 & Bachiller & Oficinista & 1. Terminalista. \\
\hline 32 & E32 & Bachiller & $\begin{array}{l}\text { Empleado } \\
\text { de aseo }\end{array}$ & 1. Ningún curso \\
\hline 33 & E33 & Bachiller & Oficinista & 1. Oficinista de comunicaciones \\
\hline 34 & E34 & Bachiller & Oficinista & 1. Único cargo desde que ingresó. \\
\hline 35 & E35 & Bachiller & Oficinista & 1. Único cargo desde que ingresó. \\
\hline
\end{tabular}

\section{Notas:}

1. Las letras sustituyen a los nombre para proteger sus identidades y porqué realmente no es de interés su identificación concreta.

2. TSU: Técnico superior universitario.

3. El pruebista se encarga de organizar la contabilidad diaria de la oficina.

4. Gobierno es el encargado de administrar las cuentas del gobierno en sus diversos niveles.

5. Banca privada es la persona encargada de atender a los clientes preferencias de depósitos o crédito.

6. El gerente de avance es un gerente itinerante, que suple las vacantes de los gerentes titulares por un período determinado.

7. Ctas. Ctes.: Cuentas Corrientes.

8. Datos suministrados por los empleados de la oficina bancaria. 
Resistencia al cambio en las organizaciones desde la perspectiva del estructuralismo... Borgucci, Emmanuel

tal manera que al final de la tarde, cuando se realiza la prueba final, la máquina arroje los resultados por cuanta movilizada de cargo y abono y así elaborar la hoja de prueba (balance de comprobación) del día.

En consecuencia, los pruebistas trabajan estrechamente con los cajeros, el cajero principal y los terminalistas. Por lo tanto, de presentarse atrasos en las operaciones de caja y/o de terminal, la prueba se atrasa.

Dentro del segundo grupo existía una gran solidaridad debido al tipo de trabajo cotidiano que realizaban. Este trabajo consistía en postear todo tipo de operaciones provenientes de los cajeros, el área de atención al público incluyendo la banca privada y el área de crédito. Además, eran los encargados de formar el equipo de pago fuera de sistema (cuando había caída de línea) y de procesar entre las 11:45 am hasta las 2:30 pm la cámara de compensación que llegaba de la oficina principal de la ciudad y que debía ser devuelta con todos los cheques procesados y con autorización o no de pago de acuerdo a las condiciones del cliente (condiciones especiales o no). La presión del trabajo provenía de la sobrecarga de documentos a procesar, las caídas de sistema y los cheques de compensación. Además, en la tarde después de cerrar las puertas al público debían cuadrar con el departamento de prueba y el cajero principal. De lo contrario, debian buscar las diferencias (cargos o abonos en exceso o defecto). Su labor concluía cuando pedían el cuadre general del terminal y este era compatible con el departamento de prueba y caja principal. Por lo general, su labor no admitía pausa y/o equivoca- ciones. Las equivocaciones consistían por lo general en abonar a cuentas incorrectas o cargar a clientes cuando en realidad debían hacerlo a otras cuentas. Las peticiones más importantes de estos empleados eran: que la oficina contara con un controlador propio y más terminalistas. Sin embargo, ellos sabían, por su experiencia, que esas peticiones no serían cumplidas. Más bien la vicepresidencia regional así como gerentes de otras agencias los consideraban como terminalistas lentos.

El grupo de cajeros es el que más relaciones tiene con el público. Ellos realizan básicamente dos tipos de operaciones: recibir depósitos y pagar cheques/retiro de depósitos. La primera labor consiste en verificar el monto de la consignación en efectivo, cheques del propio banco (de la plaza o fuera de ella) y cheques de otros bancos (de la plaza o fuera de ella) con el físico (efectivo y cheques). Además en los cheques debe verificar el endoso y establecer los bloqueos respectivos a los cheques presentados al depósito. La segunda labor consiste principalmente en verificar los cheques de acuerdo a lo que establece el Código de Comercio, los endosos y requerir los documentos necesarios en caso de ser cheques de empresas. Su labor es crítica cuando cae el sistema de transmisión de datos, debido a que se tiene que pagar bajo la modalidad de fuera de línea. Al final del día, deben realizar su cuadre de efectivo así como de pagos e ingresos que no son en efectivo. Su información debe entregarse al departamento de prueba y al de caja principal para que éste elabore el "Resumen del movimiento de caja del día". Por otra parte, de acuerdo a 
las disposiciones de seguridad bancaria, parte del efectivo de depositar fuera de la oficina y para eso se elabora una "Carta remesa" de efectivo a ser despachado al servicio de transporte de valores, un "Comprobante de servicio" especificando la empresa de valores a ser utilizada, se elabora el envase con el efectivo y se le coloca el plomo con un sello.

El grupo de personas de atención al público está conformado por personas jóvenes especialmente mujeres. Estas personas tienen poca experiencia en el manejo de las operaciones de pruebaterminal-caja. Su labor es la captación de clientes potenciales, cruzar ventas de servicios financieros y, en algunos casos, ofrecer atención personalizada a clientes de importancia. Su entrenamiento se sustenta principalmente en aprender y aplicar tácticas de mercadeo directo, atención al público y en conocer los principios que rigen los diversos productos/servicios que ofrece la institución.

El grupo de funcionarios de la oficina principalmente se asegura de que los diversos sistemas operativos de la oficina funcionen. También su labor es la supervisión, vigilancia y sanción de prácticas consideradas como irregulares desde el punto de vista de la auditoria y prácticas consideradas como al margen de la ley. Por otra parte deben dar cumplimiento a las metas asignadas a la oficina durante el semestre, con la finalidad de conseguir una buena clasificación. Desde el punto de vista de Bourdieu (2001), el gerente representa en el microcosmos de la oficina, el poseedor del capital simbólico que representa ser la autoridad y la persona que se supone que conoce todos los car- gos de la oficina. Por otra parte, los subgerentes no son solamente funcionarios encargados de las operaciones de crédito y administración. Su papel también incluye ser agentes autorizados para usar el capital simbólico que ostenta el titular de la oficina, tanto en caso de su ausencia como en las operaciones cotidianas.

El gerente de la agencia reproduce las prácticas que identifican a la organización en su conjunto y que son reflejo del grupo de gerentes que conforman la institución. En esta organización algunas de esas prácticas distintivas son:

1. Se sienten con autoridad de seleccionar su clientela

2. Tienen autoridad de seleccionar su personal de confianza.

3. Pueden determinar la carrera de algún empleado de acuerdo a la apreciación que el gerente tenga de esa persona.

4. Establece las estrategias para alcanzar las metas.

5. Utiliza sus relaciones personales para promoverse dentro del grupo de gerentes.

6. Puede formar equipos de trabajo que puede movilizarse al ser ascendido.

7. Impone el ritmo de trabajo en la oficina.

8. Según su relación con los clientes, establece el esquema de atención que los empleados deben tomar en consideración.

9. Puede participar en la elaboración de los objetivos del semestre en materia de colocaciones y captaciones.

10. Administración de la información. Puede hacer que los empleados de atención al público o los cajeros recojan ciertas informaciones de los clien- 
Resistencia al cambio en las organizaciones desde la perspectiva del estructuralismo... Borgucci, Emmanuel

tes que se habían menospreciado anteriormente (Badoc, 1975: 230).

11. Organiza el mercadeo directo y sus relaciones públicas.

12. Administra el personal. Como presidente de la oficina, asume el papel de gerente de recursos humanos y él es el mejor medio de promoción de empleados a mejores cargos.

En resumen, el gerente y los demás funcionarios de la oficina bancaria han ejercido un poder estructurador sobre las relaciones entre los diferentes grupos de personas que conforman la nómina de la agencia por medio de la puesta en circulación de una serie de símbolos, historias y narraciones (Bourdieu, 2001: 92). Este poder se manifiesta, cuando está consolidado, en una aceptación o adhesión natural del sentido de los procesos operativos, administrativos y comunicativos que identifica la labor desarrollada en la agencia. Es decir, se presenta una convergencia de voluntades sustentada en el convencimiento de la necesidad de que la labor realizada tiene un sentido en términos organizacionales (la organización impone una trayectoria a seguir), de remuneraciones y promoción dentro de la estructura jerárquica.

Ahora bien, en esta oficina el proceso de promoción o ascenso en una oficina bancaria comienza por lo general por ser empleado de atención al público, pasando luego por crédito, los puestos de prueba, terminales y cajero. Después de cajero, el empleado puede optar, de acuerdo a su desempeño, a ser promovido como funcionario (con la obtención de la firma numerada) de atención al público y posteriormente a ser subgerentes de crédito, de administración y finalmente gerente de oficina. Para ser nombrado gerente de oficina, se requiere un periodo de prueba que puede alcanzar hasta los tres años. En ese período el funcionario recorre diferentes oficinas de la región donde presta servicios con la finalidad de conocer la clientela del banco en diferentes sub-regiones (los Andes, Zulia o Falcón). Por otra parte, comienza a conocer diferentes sistemas de trabajo y grupos de empleados en las diferentes oficinas. Luego de terminar ese periodo de entrenamiento empírico, el nombramiento definitivo va a depender, entre otras cosas de: algún gerente activo se jubile, reorganización de la oficina, promoción de un gerente existente o apertura de una nueva oficina.

Ahora bien, entre los desafíos que debe enfrentar esta plana de gerentes son:

1. Atender la sobrecarga de trabajo de los terminalistas sin necesidad de ampliar su nómina.

2. Trabajar con una oficina con solamente dos (2) líneas telefónicas y una línea muerta para transferencia de datos.

3. El descontento que expresa el personal por las frecuentes caídas del sistema.

4. El descontento de la clientela por el servicio que presta la oficina.

5. El proceso de cuadre de la oficina se realiza a entradas horas de la noche y lo que es peor no se puede realizar. Eso obliga a acudir a otras agencias con controladores de datos los fines de semana para procesar toda la información del día o de la semana.

Las relaciones de grupo que se han encontrado en la observación son las siguientes: 
1. Los terminalistas y los cajeros son los grupos que lideran el tono de las relaciones laborales con los funcionarios. En otras palabras, son la mayor fuente de quejas y reclamos dirigidos a los funcionarios; cuestionan la capacidad de la gerencia para resolver problemas estructurales de la agencia como, por ejemplo, la búsqueda de la asignación de más personal y la instalación de nuevas líneas de comunicaciones.

2. Dentro del grupo de los cajeros, se encuentra el asistente del cajero principal que es el Presidente del Sindicato Nacional del Banco.

3. Por otra parte, dos de los cajeros han manifestado su intención de avanzar a cargos de supervisor. Sin embargo, están de acuerdo con el sindicato.

4. El resto de los grupos manifiestan su apoyo a los cajeros y al sindicato debido a la imposibilidad de la gerencia en mejorar sus condiciones operativas de trabajo.

5. Para estos trabajadores, lo más importante es que la oficina tenga un sistema en línea en tiempo real y que funcione de manera continua.

6. La solicitud de contratación de mayor número de personas para laborar en la agencia.

De acuerdo a lo anterior, cualquier tentativa de cambios se enfrentó a una resistencia implícita y explícita por parte de los trabajadores. Esa resistencia se manifestó en:

1. Incapacidad de la gerencia en aplicar cambios debido a que no han podido lidiar con los problemas existentes.

2. Consideran que nuevos cambios significarán más trabajo con igual salario.
3. En una reducción en el número de operaciones por trabajador por día.

4. Reducción en el número de clientes de crédito y la no incorporación de nuevos clientes.

5. Los funcionarios se convirtieron en portavoz de las exigencias de los trabajadores en la Vicepresidencia Regional.

6. Los trabajadores empezaron a demandar ante el sindicato cualquier atraso en el pago de horas extras y bonos del contrato colectivo.

7. Se manifestó, sobre todo en el departamento de prueba, de un desorden contable respecto a los movimientos de operaciones diarias.

8. El sindicato y su presidente se convirtió en el refugio de las quejas de los trabajadores. Por su parte, el sindicato asumió una postura de buen padre de familia

9. Postura reactiva de la mayoría de los empleados ante solicitudes de los gerentes.

10. Aumento de errores en la activación de material de uso controlado para los clientes (libretas de ahorro, chequeras, planillas de depósito, etc.). El informe de auditoria administrativa señaló problemas en el control de los gastos operativos directos; con el control del mobiliario activo; partidas pendientes por cruzar en las cuentas que manejan las diferentes dependencias de la agencia; falta de control en el material de uso controlado; retardo en la entrega de tarjetas de débito y crédito; problemas en la apertura y cierre de cuentas de depósitos a la vista; retardos con las cobranzas del país cheques al cobro, plazo fijo, 
Resistencia al cambio en las organizaciones desde la perspectiva del estructuralismo... Borgucci, Emmanuel

entrega de chequeras; o de la autorización de girar sobre diferido sin condiciones especiales.

11. Falta de concentración a la hora de activar el plan de pago fuera de línea. Dos ejemplos pueden ilustrar esta situación. En primer lugar, la conformación de un cheque con una firma que correspondía pobremente con el espécimen de firma microfilmado de los archivos lo que costó un retiro indebido de más de seis cifras medias. Por otra parte, se encuentra el pago de retiros de ahorro fuera de línea en que se verificó posteriormente montos abonados equivocadamente y posteriores retiros por el monto depositado por parte del cliente.

12. Por otra parte, se encuentra los desordenes en los archivos de los clientes. Esto es una labor que por su sencillez no requiere de una supervisión estricta del funcionario.

13. Se notó una especie de postura fatalista ante los problemas operativos de la oficina; es decir, que las cosas no van sino a empeorar en lugar de mejorar. Esta actitud podía llevar a que los trabajadores solamente se dedicaran a su trabajo y a cumplir su horario. Sin embargo, vieron la oportunidad de aprovecharse de la situación por medio de las horas extras. En la institución el horario de trabajo era hasta las seis de la tarde y después de esa hora hasta las seis de la mañana se pagaba horas extras. Como consecuencia de lo anterior, el ritmo de trabajo se disminuyó desde el momento del cierre al público hasta las siete y media de la tarde cuando optaban por irse a casa, sabiendo que a partir de la seis de la tarde comienza el pago de las horas extras completas nocturnas aunque se trabajara una hora. Esta práctica llevó a que los ingresos personales por horas extras fueran casi iguales al salario promedio por trabajador.

14. Se formaban largas colas en las taquillas de los cajeros al momento de comenzar las operaciones del día, a las 10:45 am y a las 3:00 pm.

15. Se formaban largas colas en la taquilla externa de la oficina (taquilla habilitada cuando la oficina no laboraba en el horario bancario) especialmente los días sábados y domingos.

16. En un estudio realizado, se descubrió que las agencias de esta institución bancaria con más de 30 empleados tienen un promedio de operaciones por día por trabajador inferior que aquellas que cuentan con menos de 20 empleados.

17. En términos de metas semestrales, esta agencia quedó descalificada debido a que no cumplió con las metas de captaciones y colocaciones asignadas en tres semestres consecutivos. Lo anterior se manifestó en el cierre de cuentas por parte de clientes importantes y la cancelación de pagarés comerciales también de clientes importantes.

En conclusión, los trabajadores estaban cuestionando la incapacidad de la empresa y de los funcionarios para llevar a cabo los cambios por ellos reclamados. Por su parte, la empresa consideraba que la labor desempeñada por los empleados y funcionarios de la agencia era deficiente y lo que estaba ocurriendo era una lucha por desplazar a la actual administra- 
ción de la agencia por las personas que por razones de experiencia deberían ser promovidos a cargos de funcionarios. Según la gerencia regional, esa pérdida de control por parte de los funcionarios era el comienzo de la implantación de un nuevo esquema de trabajo liderado por los empleados y amparado por el sindicato nacional del banco. Esta afirmación se sustentaba en que si bien era necesario ajustes operativos estos no deberían seguir el modelo de la empresa sino uno diseñado desde los empleados y refrendado por el sindicato. Esta afirmación se correspondía a un modelo en el que los empleados pasarian a ser los nuevos funcionarios pero con mentalidad de empleados. Es decir, pasar de un modelo con separación de intereses propio de la lógica del funcionario a una lógica de confusión de intereses empleado-funcionario. El segundo modelo es favorable al empleado debido a que garantiza que cualquier decisión deberá hacerse tomando en consideración otros criterios. Alguno de estos criterios serían: evitar despidos en el personal, aumentar la nómina, luchar por la sindicalización de los funcionarios o mejorar las condiciones de la contratación colectiva hasta la búsqueda de una administración conjunta.

Ante la situación descrita, la dirección del instituto bancario estaba en la disyuntiva de dejar las cosas como estaban o decidir el cierre definitivo de la agencia. La alternativa que se eligió fue la de no cerrar la agencia, pero someter a prueba al personal para una posible mudanza de la agencia. En tal sentido se procedió en dos etapas. En la primera etapa se decidió realizar dos cambios: sustituir progresivamente al equipo ac- tual de funcionarios, e incorporar a la agencia en el sistema de "Nueva Oficina Bancaria", con el sistema denominado "Plataforma Bancaria". En la segunda etapa, realizar la selección definitiva de personas para la nueva ubicación de la agencia, con un nuevo cuerpo de funcionarios y nueva infraestructura técnica de servicios.

La primera etapa fue la más traumática por dos razones. En primer lugar, el nuevo cuerpo de funcionarios era totalmente desconocido por los empleados y provenían de Caracas, que es una plaza en donde el volumen de presión en el trabajo es mucho más elevado. En segundo lugar, se rompió la pretensión implícita o explícita de los empleados de aspirar a implantar una nueva lógica de gestión en la agencia.

La presión en el trabajo se incrementó debido a que los nuevos funcionarios trataron de aplicar con rigor las normativas operativas en las diferentes áreas de la agencia sin que eso implicase pagar más horas extras de las obtenidas en el pasado. Por otra parte, todo tipo de operaciones era revisada exhaustivamente, se incrementaron los controles sobre el material de uso controlado, se pidió un mayor número de cuadres de la agencia, se realizó mayores arqueos a los cajeros. Esas medidas iniciales provocaron una serie de situaciones reconocidas como resistencia al cambio: Ios funcionarios actuales manifestaron su preocupación por el clima laboral de la agencia y su posición a favor de los empleados, algunos empleados manifestaron abiertamente su contrariedad con el trato dado por los nuevos funcionarios, se presentaron conatos de protestas de los empleados de caja y terminales, y aumento 
Resistencia al cambio en las organizaciones desde la perspectiva del estructuralismo... Borgucci, Emmanuel

del número de reclamos al sindicato del banco entre otras situaciones.

Por ello, la Dirección Regional del Banco se propuso realizar un intercambio de funcionarios actuales de la agencia hacia la oficina principal ocupando el mismo puesto. Al mismo tiempo se trajeron funcionarios de la sucursal para laborar en esta agencia. Por otra parte, también se elaboró un plan de rotación de personal de la agencia hacia otras oficinas y traer empleados de otras oficinas por un tiempo de tres meses. Estos cambios garantizaron, según mi entender, reestablecer el control sobre el número y calidad de las operaciones realizadas y reestableció la lógica de la empresa (habitus) en el manejo de situaciones críticas sobre todo fuera de línea. En tal sentido se optó por utilizar, en determinadas horas del día, una línea telefónica básica para transferir datos. Esta idea tuvo un impacto en el aumento del número y continuidad de las operaciones en tiempo real.

Por otra parte, se diseñó un plan de recuperación de clientes con el Departamento de Crédito que consistió en: recuperar los clientes tradicionales perdidos por medio de visitas a las empresas y clientes personas naturales y un plan de captación de clientes potenciales por medio del diseño de un plan de mercadeo directo, elaborando un catastro de empresas y personas naturales en que cada semana se cubriría seis manzanas a la redonda de la agencia para establecer los límites de su "zona de influencia". Por otra parte, para agilizar las aperturas de cuentas, uno de los funcionarios saldría a la calle con uno de los empleados de atención al público para abrir en el lugar las cuentes que sean necesarias.
Los resultados en el primer semestre fueron: un aumento en el número de clientes de crédito y depósitos, un aumento significativo en el número de operaciones por empleado por día, un aumento en el número de cheques de cámara de compensación local y nacional procesados, una reducción de los días sin sistema de comunicaciones en línea y en definitiva una clasificación de tipo B. Lo más importante es que se reestableció el control gerencial, en la medida en que pusieron en circulación nuevamente un discurso que enfatiza los valores de la disciplina en el trabajo, el respeto a la jerarquía, las normas de seguridad y lo que caracteriza a un banquero conservador. Esto permitió que ciertas actitudes hayan obtenido el reconocimiento para estar en condiciones de imponerse por sobre otras actitudes y representaciones, debido a su capacidad de adecuarse a las necesidades de los grupos en disputa, se incrementa su eficacia simbólica.

La segunda etapa ocasionó un nuevo nivel de tensiones entre los funcionarios y los empleados, debido a que la transformación de los sistemas operativos implicaba pasar de ser especialistas en una sola área a ser especialistas en múltiples áreas. Es decir, un empelado de atención al público tendría a su cargo una estación de trabajo en donde desempeñaría actividades de cajero, pruebista, terminalista y vendedor; mientras que un antiguo cajero debería desempeñar actividades que antes correspondían al empleado de atención al público. En el primer caso, la persona debería trabajar con dinero físico y conocer los códigos de transacción de las operaciones de posteo del terminal. En el segundo caso, la per- 
sona debe dominar todos los procedimientos necesarios en las operaciones de apertura y cierre de diferentes instrumentos de colocación y de captación. Lo más grave fue que el curso solamente tuvo una duración de 12 horas entre los días sábado y domingo, debido a que el día lunes después del curso comenzaba a funcionar el nuevo sistema. Sin embargo, la nueva gerencia sentía contar con más control para administrar las diversas fuerzas que atentaban contra el logro del objetivo de implantar el nuevo sistema operativo.

La última fase de reestructuración de la organización en el ámbito de esta agencia consistió en seleccionar el personal que sería asignado a la nueva oficina. Es curioso destacar que ningún empleado fue despedido en ese traumático proceso y más bien fueron todos seleccionados para laborar en la nueva oficina y quienes estaban en la lista para ser promovidos al cargo de funcionarios fueron seleccionados. Es decir, se siguió la rotación de puestos y se demostró que la organización retomó el control y dominio de las prácticas administrativas y operativas que le son propias.

\section{Algunos planteamientos para finalizar}

Los procesos administrativos que se presentan como lógicos o naturales dentro de una organización son realmente construidos por las interacciones entre los diferentes grupos que participan. Sin embargo esa participación es asimétrica en donde existen personas pertenecientes a un grupo poseedor del capital simbólico o administrativo que les permite fijar las re- glas en las que otros deben adherirse. Esa condición de pertenencia genera una creencia o poder motivador de quienes no son el grupo líder para alcanzar el sitial de poseedor del capital simbólico, económico o administrativo. En el proceso, las personas desarrollan estrategias y tácticas dentro de los límites de las reglas de juego establecida por los funcionarios y la institución, generando una serie de prácticas administrativas y gerenciales admisibles y discursos posibles.

Sin embargo, la experiencia demuestra que quienes tienen el monopolio del capital gerencial se exponen a ser desafiados y que la resistencia al cambio puede contribuir a socavar el control y dominio que anteriormente ejercía determinado equipo de funcionarios. Además, se observa que en organizaciones como los bancos las reglas de adhesión se están incrementando en dificultad cada vez que se incorporan nuevos avances tecnológicos y que se pone más interés en un empleado bancario operativamente eficiente y efectivo como vendedor. En tal sentido es pertinente la cita de Bourdieu (1996) en donde cuanto más peligrosa es una situación más codificada tiende a ser su práctica.

\section{Referencias bibliográficas}

Badoc, Michel (1975). Marketing en los bancos europeos. Editorial Tecniban. Madrid. Pp. 338.

Barquero, José Daniel; de Llauder, Carlos Rodríguez; Huertas, Fernando; y Barquero Mario (2003). Marketing de clientes. Editorial McGraw Hill/Interamericana de España, S. A. U. Madrid. Pp. 291.

Bourdieu, Pierre (1996). Cosas Dichas. Editorial Gedisa, S. A. Barcelona. 
Resistencia al cambio en las organizaciones desde la perspectiva del estructuralismo... Borgucci, Emmanuel

Bourdieu, Pierre (1999). ¿Qué significa hablar?: economía de los intercambios lingüísticos. Akal ediciones. Madrid.

Bourdieu, Pierre (2001). Poder, Derecho y Clases Sociales. Editorial Desclée de Brouwer, S. A. Bilbao. Pp. 232.

Brown, Marvin. T. (1992). La ética en la empresa: estrategias para la toma de decisiones. Primera edición en castellano. Ediciones Paidós Ibérica, S. A. Barcelona. Pp. 278.

Burns, T y Stalker, G.M. (1961). The management of innovation. Tavistock. London.

Cáceres, Galindo Jesús (1998). Técnicas de investigación en sociedad, cultura y comunicación. Primera reimpresión. Addison Wesley Longman. Naucalpán de Juárez. Pp. 523.

Foucault, Michel (1997). Vigilar y Castigar. Nacimiento de la prisión. Vigésimo séptima edición en español. Siglo XXI editores, S. A. de C. V. México. Pp. 314.

Gutiérrez, Alicia B. (2002). "La lógica del juego. La noción de campo en la perspectiva de Pierre Bourdieu". En Trayectorias. Año 4. No. 10. Septiembre-Diciembre. Santa fe de Bogotá D. C. Pp. 9- 19.

Hill, W.L. Charles y Jones, R. Garret (1996). Administración estratégica: Un enfoque integrado. Editorial McGraw Hill/Interamericana, S.A. Santafé de Bogotá. Pp. 540.

Hofer, Charles W. y Schendel, Dan (1985). Planeación estratégica: Conceptos analíticos. Editorial Norma, S. A. Sta. Fe de Bogotá. Pp. 228.

Hurtado de Barrera, Jacqueline (1998). Metodología de la investigación holística. Tercera edición. Fundación Sypal. Caracas. Pp. 630.
Kotler, Philip (1996). Dirección de mercadotecnia. Octava edición. Pretince-Hall Hispanoamericana, S. A. Naucalpán de Juárez. Pp. 800.

Lambin, Jean-Jacques (1995). Marketing estratégico. Editorial McGraw Hill/Interamericana de España, S. A. U. Madrid. Pp. 610.

Lashinsky, Adam (2006). "Chaos by design". En Fortune, Europe edition. No. 17 Pp. 34-42.

Leana, Carrie y Rousseau, D. (2000). Relational wealth: The advantages of stability in a changing economy. Oxford University Press. New York.

Leana, Carrie y Van Buren (1999). "Organizational social capital and Employment practices". En Academy of Management Review. Volumen 24. Pp. 538555.

Leana, Carrie y Barry Bruce (2000). "Stability and change as simultaneous experiences in organizational life". En Academy of Management Review. Volumen 25. No. 4. Pp. 753-759.

Martínez, Miguel (1989). Comportamiento humano. Nuevos métodos de investigación. Editorial Trillas, C. A. de C. V. México, D. F. Pp. 307.

Mishkin, Frederic S. (1995). The Economics of money, Banking and Financial Markets. Fourth Edition. The Harpers Collins Colleges Publishers. New York. Pp. 758.

Norbert, Elias (1995). Mi trayectoria intelectual. Primera edición. Provenòa. Barcelona.

Pfeffer, J. (1981). Power in organizations. Edit. Pitman. Boston, Ma.

Pfeffer, J. (1998). The human equation: Building profits by putting people first. Harvard University Press. Cambridge, MA.

Robbins, P. Stephen (1987). Comportamiento organizacional: Conceptos, con- 
troversias y aplicaciones. Tercera edición. Editorial Pretince Hall Hispanoamericana, S. A. Naucalpán de Juárez. Pp. 566.

Robbins, P. Stephen y Judge, Tim (2006). Organizational Behavior. Pretince Hall. Pp. 688.

Sennett, R. (1998). The corrosion of character: The personal consequences of work and the new capitalism. Editorial Norton. New York.

Stoner, A.F. James y Wankel, Charles (1989). Administración. Tercera edición. Pretince-Hall Hispanoamericana, S. A. Naucalpán de Juárez. Pp. 826.

Townley, Barbara (1993). "Power/Knowledge, and its relevante for human resourse management". En Academy of Management Review. Vol. 18. No. 3. Pp. 518-545.

Vassos, Tom (1996). Estrategias de mercadotecnia en Internet. Pretince-Hall Hispanoamericana, S. A. Naucalpán de Juárez. Pp. 325.

Williamson, O. (1975). Markets and hierarchies. Free Press. New York.

Woodman, W. Richard, Sawyer, E. John y Griffin, W. Ricky (1993). "Toward a theory of organizational creativity". En Academy of Management Review. Vol. 18. No. 2. Pp. 293-321. 